\title{
Applying Virtual Environments to Manufacturing
}

\section{Sandy Ressler}

U.S. DEPARTMENT OF COMMERCE Technology Administration National Institute of Standards and Technology

Gaithersburg, MD 20899 



\section{Applying Virtual Environments to Manufacturing}

\section{Sandy Ressler}

U.S. DEPARTMENT OF COMMERCE Technology Administration National Institute of Standards and Technology

Gaithersburg, MD 20899

January 1994

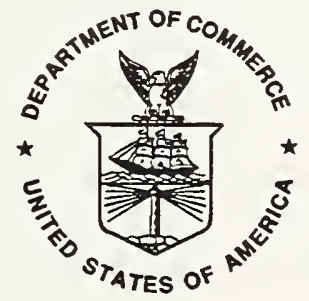

U.S. DEPARTMENT OF COMMERCE Ronald H. Brown, Secretary

TECHNOLOGY ADMINISTRATION

Mary L Good, Under Secretary for Technology

NATIONAL INSTITUTE OF STANDARDS

AND TECHNOLOGY

Arati Prabhakar, Director 



\title{
DPEN Applying Virtual Environments REALITY to Manufacturing
}

\author{
Sandy Ressler \\ National Institute of Standards and Technology
}

\begin{abstract}
When we first announced this hearing the Commerce Committee office got dozens of telephone calls from people asking what is "virtual reality" and why are you holding a hearing on it? Well, the answer is fairly simple. Virtual reality promises to revolutionize the way we use computers. At least that's my opinion. It could improve the way we design new products, how we teach our children, and how we spend our free time. It has the potential to change our lives in dozens of ways.
\end{abstract}

Albert Gore opening statement at May 1991 Committee on Commerce, Science and Transportation United States Senate Hearing.

\subsection{Virtual Environments - Some Background}

Virtual environments (VE), altemately known as virtual reality (VR), synthetic environments and other names, are a collection of technologies which, offers the opportunity to integrate the human into a computing system. The technologies which are used to create a virtual environment are: a high speed graphics display computer, a head mounted display, a 3D position tracker/input device, and spatial audio. When combined into a single system the computer application can track a user's head position and generate a display that changes depending on where the user is looking. The 3D input device allows the user to interact with synthetic objects and the spatial audio heightens a user's sense of place.

For example a user could interact with a virtual lathe. The lathe, actually a computer graphics image, spins on the display screen and the user moves a virtual cutter with a 
3D pointing device [Sun 92]. Additionally users can sit in the cab of a yet-to-be-built piece of equipment and survey the visual obstructions.

User interfaces, the ways in which we use computers, are in general a bane to many users. By and large, computers are too difficult to use, and a principal reason is poor user interfaces.

Technical experts, for example, a machinist or shop foreman, are forced to become computer experts, generally against their will, not because of an interest in computers but because of the obtuse nature of the computer program they need. Virtual environments offer the potential of placing the user inside the data. Rather than learning an obscure control language in order to operate a simulation one will be able to simply reach out and push the data around. Of course, the technologies of virtual environments are not quite ready for prime time, however it is also clear that steadily improving hardware and software will provide adequate performance at reasonable prices.

\subsection{Case Studies}

Even though virtual environment technologies are still difficult and expensive to use, people are doing real work. The following case studies represent examples of recent state of the art work that exemplifies the application of virtual environments in one or several aspects of manufacturing. Manufacturing, in this case, is taken to encompass issues relating to maintenance and training as well as the actual creation of parts and the assembly of systems. These examples are actual real world systems, not simply speculative fantasies.

\subsection{Boeing}

The Research and Technology organization of Boeing Computer Services is actively pursuing two projects using VR technology. According to David Mizell, manager of Virtual Systems Research \& Technology, Boeing uses a concept known as Augmented Reality.

Augmented Reality is a term which refers to the ability to see through a computer generated display. The generated images are superimposed on top of reality. This is accomplished by projecting a computer image onto a half silvered mirror that the user looks through. This technique provides an effective and intuitive way of "annotating" reality. The Boeing team is using a head set configured for augmented reality, which they call a HUDset (Heads-Up, see-through, head-mounted Display).

The assembly of aircraft is a highly complex task that is difficult to automate. Many of the skills required demand dexterity not easily accomplished by robots. In addition, airplanes consist of many small-lot size parts and reprogramming robots for these quantities is an expensive prospect. To quote from Caudell and Mizell's paper [Caudell \& Mizell 1992] "Someone once said that a Boeing 747 is not really an airplane, but five million parts flying in close formation." The illustration below shows how a pop-up 
annotation points out the location for a drilling operation. The annotation appears to float in the air on top of the actual drilling site.

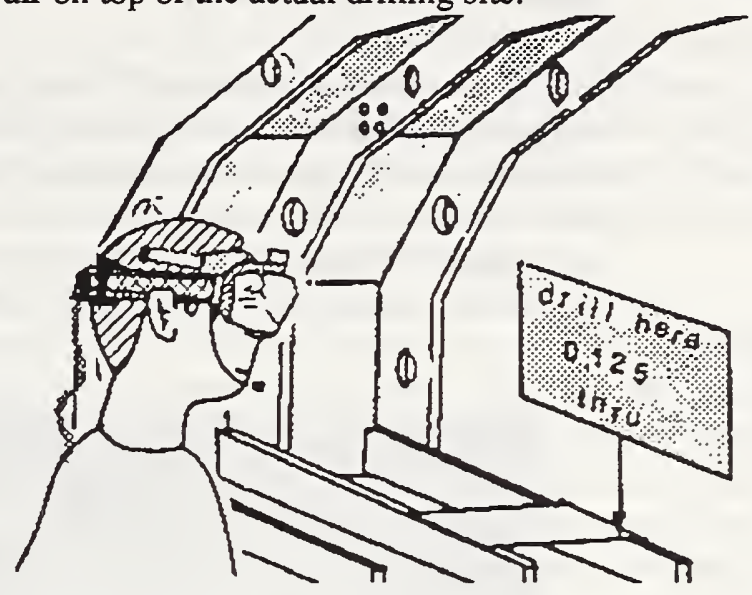

FIGURE 1.

Boeing HUDSET (heads up display see through head mounted display)

The particular task being addressed by Boeing's AR system is the assembly of wire harnesses. To quote again from Caudell and Mizell's paper:

The first of these is the wiring formboard, where wire bundles are manufactured by laying individual wires across pegs on an annotated board. In today's factory, the board is annotated with a long, computer-generated plot of the wire bundle glued to sheets of plywood. The assembly worker must be able to read the paper drawing as the bundle grows. For this demonstration, a blank peg board represents the formboard. The computer indicates the position of the pegs for the current wire bundle assembly task for the user to install. After this, the paths of individual wires are indicated sequentially on the board as a series of bright red lines.

The second application is connector assembly. After the wire bundle is assembled, the ending leads are inserted into a multipinned connector. The factory worker must consult a paper "map" of assignments, telling what wire goes into which pin. For the larger connectors, as many as fifty wires are mapped to pins, an error-prone task. In this demonstration, a red indicating line appears to protrude from the connector at the exact location where the current wire is to be inserted. A text window tells the user the number and description of the correct wire. The user moves to the next wire by again hitting the space-bar on the keyboard.

\subsection{Caterpillar}

Researchers at Caterpillar Inc. have used VR to improve the design process for heavy equipment. Dave Stevenson and John Bettner engineers with Caterpillar in collaboration with the staff of NCSA (National Center for Supercomputing Applications) have 
put together a system which allows them to quickly prototype wheel loader and backhoe loader designs [NCSA 1993].

In particular, the team is able to perform visibility assessment of the new design. Engineers put on a helmet-mounted display and have a full 360 degrees of vision to see how the environment looks and to evaluate obstructions. A Silicon Graphics computer is used to generate the real time graphics display and to simulate the operation of the equipment. The engineers can "operate" the equipment and evaluate visual obstructions in a natural manner without having to build a physical prototype.

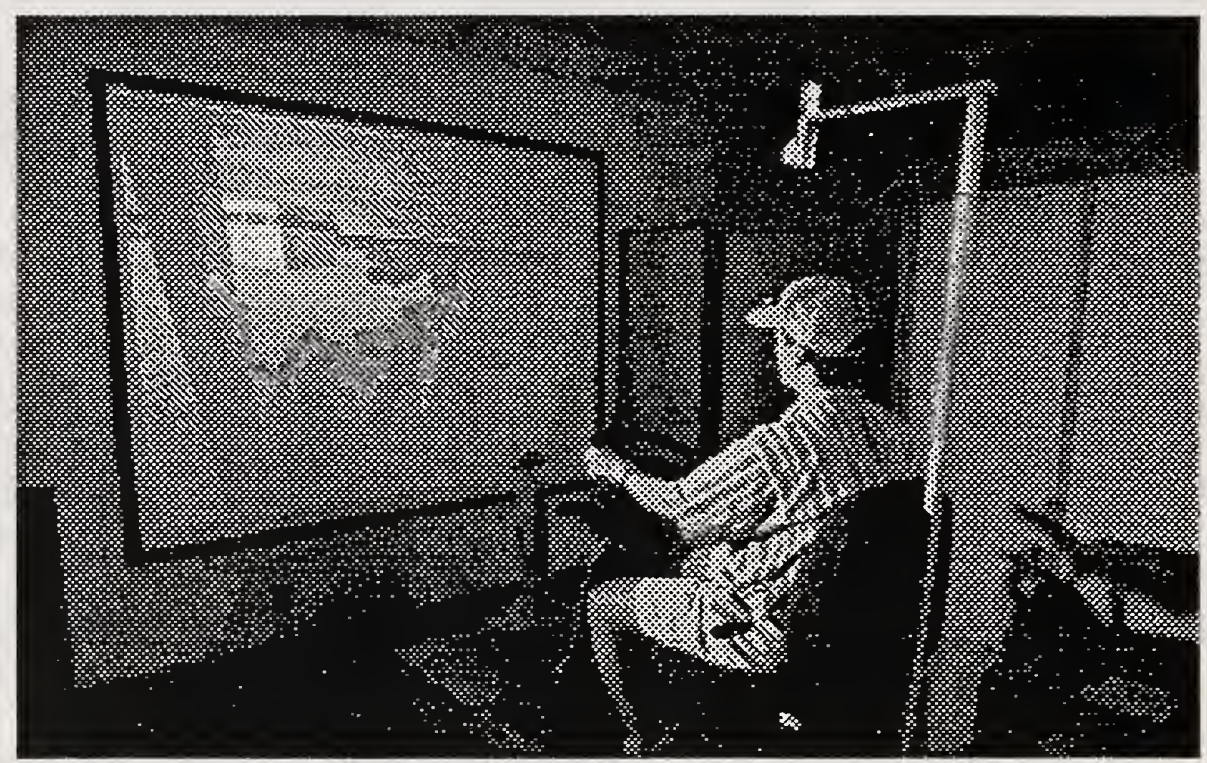

FIGURE 2.

This image from the Virtual Backhoe project illustrates an "operator" driving the virtual equipment at the NCSA VR lab.

The Caterpillar team was awarded the 1993 NCSA Industrial Challenge Award for VR Use. The press release announcing the award stated:

This technology allows us to dramatically shorten the amount of time it takes to analyze a new design concept and incorporate it into our production process," design Engineer Dave Stevenson said. "It also represents a sizeable cost savings because we aren't having to build prototype machines or make last-minute design changes." He said it takes six to nine months to build fullscale models and design changes using conventional design methods. However, using the virtual reality approach, designs usually can be evaluated in less than one month. Company officials said a number of design options already been tested for new models of wheel loaders and backhoe loaders that are to be introduced by 1996, and the company said it eventually plans to allow customers to "field test" new products by putting on the special helmet. 


\subsection{Ford}

Within a development organization called Ford Alpha Simultaneous Engineering there is an evaluation of VR for automotive assembly taking place. According to Jim Merner, [Merner 1992] manager of the VR project, they are evaluating process installation feasibility. The vehicle parts are represented in a CAD system. The CAD file is transferred to the system with the VR equipment. A user then manipulates the virtual part and attempts to assemble it into the virtual vehicle.

The equipment used for the VR experiments are a VPL eyephone and dataglove running on a Silicon Graphics computer. The user puts all the equipment on and attempts the part insertion. The system checks for interference and collision between the part and the vehicle. The hope is to use the VR setup to evaluate the human ergonomics of various assembly operations. Eventually they plan to place some more motion trackers on the person to evaluate how much bending and stooping is necessary to complete the assembly.

\subsection{Matsushita's Virtual Kitchen}

One of the most widely publicized examples of VR used by the public is a setup created by Matsushita in Japan. To quote from Newquist's article [Newquist 1993]:

The most famous (and in danger of becoming something of a self-caricature) is Matsushita's Virtual Kitchen, a retail application set up in Japan to help people choose appliances and furnishings for the relatively small kitchen apartment spaces in Tokyo. Users bring their architectural plans to the Matsushita store, and a virtual copy of their home kitchen is programmed into the computer system. Buyers can then mix and match appliances, cabinets, colors, and sizes to see what their complete kitchen will look likewithout ever installing a single item in the actual location.

The Matsushita VR Kitchen is significant because it one of the few examples of a VR system set up for public use that is not a game or in a research lab. The general public is invited to use the configuration.

\subsection{Equipment Maintenance - Columbia University}

Some interesting work in the use of augmented reality for equipment maintenance is being done at Columbia University under the direction of Steven Feiner [Feiner, Macintyre \& Seligmann, 92]. The testbed application for this project is end-user laser printer maintenance. The system configuration consists of a Reflection Technology Private Eye, Logitech 3D position trackers and a mirror beam splitter. Although the scenario used at Columbia is simplistic their innovation lies in coupling a knowledge based system with the AR configuration. Their system IBIS (Intent-Based Illustration System) is a rule-based system that designs illustrations, according to a prioritized list of communicative goals. What this means is that the system plays an active role in determining where and how information is conveyed to the user. This reduces the amount of effort needed to create a specific model of the virtual world. The user is shown only the infor- 
mation determined to be relevant to the specific task at hand, and at the appropriate time.

During operation of a paper filling operation, a vector graphic display is superimposed on top of the real object. The toner cartridge is highlighted with dotted lines and the paper tray is highlighted with a textual callout.

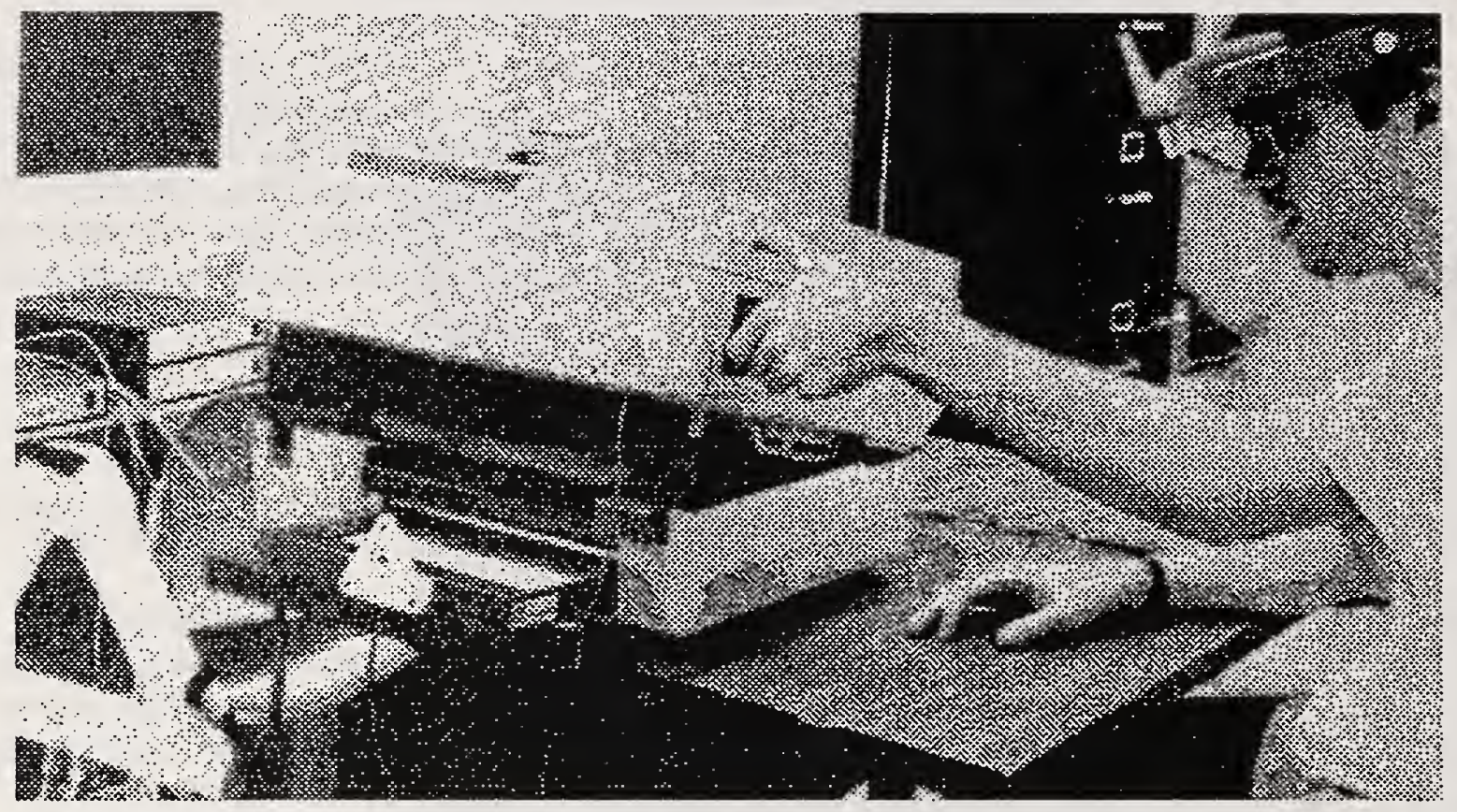

\subsection{Maintaining Equipment in Space}

The Software Technology Branch of NASA's Lyndon B. Johnson Space Center is exploring the use of virtual environment as a training aid. Maintenance of the Hubble Space Telescope as well as Space Station Freedom is difficult. How do you train astronauts to repair equipment in the weightlessness of space with realistic dynamics of the objects? Virtual environments which immerse the user into a synthetic space can play an important role in training for such maintenance missions. [Loftin 93]

In an attempt to address these issues two research and development projects are underway. The objectives of the Hubble Space Telescope Repair and Maintenance are:

To develop a Virtual Environment suitable for training astronauts in preparation for Hubble Space Telescope Repair and Maintenance missions. Graphical models of the Hubble Space Telescope (HST) and a large number of digitized close-out photographs of the instrument are available. A virtual environment based on a graphical model of the HST is under development. Where high fidelity, detailed images are needed, 
appropriate digitized images will be displayed. The system is designed to address specific elements of future missions that will require location of objects and interaction with handholds, panels, fasteners, and orbital replacement units.

A second demonstration project, Training for EVA (Extra Vehicular Activity) Payload Interaction has as its stated objective:

To develop a proof of concept training environment that provides astronauts with a simulation of satellite dynamics that match those observed during the STS-49 Intellsat retrieval mission. If the proof of concept system is judged to be valuable for training by training personnel and experienced astronauts, a more complete model will be developed and delivered as a training system for future payload retrieval missions.

As was observed in the 1992 rescue of the STS-49 Intellsat satellite, the capture and manipulation of large objects in a weightless state is quite difficult. It is hoped that the use of virtual environments will give crew members an opportunity to develop and be trained on new procedures. According to Bowen Loftin, manager of the program, the initial demonstration proof-of-concept of a training environment will operate as follows:

The objective of this demonstration is to allow users to experience a training session in an interactive virtual environment by exchanging a faulty battery in the Hubble Space Telescope (HST) with a new replacement battery. The user moves to the HST, opens the door to the battery bay, takes it back to the space shuttle orbiter, and places it on top of the hardware pallet in the payload bay. The user than takes the new battery from the cargo bay hardware pallet to the HST and installs it behind the battery bay door. A narrator will introduce a few details to the user in the beginning of the session, and correct the user when he/she makes a wrong move. The user can grab the bay door or the battery by reaching out and clinching the yellow handle with the gloved hand. Movement about the environment is accomplished with the two hand-controllers of the chair. This chair emulates the functionality of a Manned Maneuvering Unit and uses a translational control (left side) to move in any straight line by pulling or pushing in the desired direction of motion. Also, a rotational control (right side) adjusts the user's orientation about his/her center point. Changes are initiated in yaw by twisting the control left or right, in pitch by pulling it back or pushing forward, and in roll by tilting it left or right.

Benefits using a distributed virtual environment are also anticipated. The Software Technology Branch is collaborating with the Marshall Space Flight Center to allow users at the two centers to share the same virtual environment. Using these techniques, joint training exercises could be conducted without the usual collocation constraints. 


\subsection{Prototyping Analysis}

A pioneering application of virtual reality was the NASA Windtunnel. A 3D workstation generates flow field visualizations. The user interacts with the simulation via a BOOM display and a data glove. (A BOOM is a video display counterbalanced on a boom support which the user easily moves with their head.) The data glove effectively acts as a source of "smoke," allowing the user to observe local flow lines. The computation of unsteady flow visualization occurs in real time and the user can view the dynamic scene from many points of view and scales. According to a description of the system [Bryson, Levit 92]:

Since fluid dynamic phenomena occur over a large range of scales (several orders of magnitude in space), navigation through the virtual environment is more difficult than, say, architectural walkthrough. Thus, in addition to "standard" viewing capabilities sensitive to head position and head orientation, our virtual environment lets the user rapidly change his or her scale, or the scale of the environment. Relative to the environment, a user can shrink to become completely surrounded by some small vortex or grow so that the entire flow field fits in one hand.

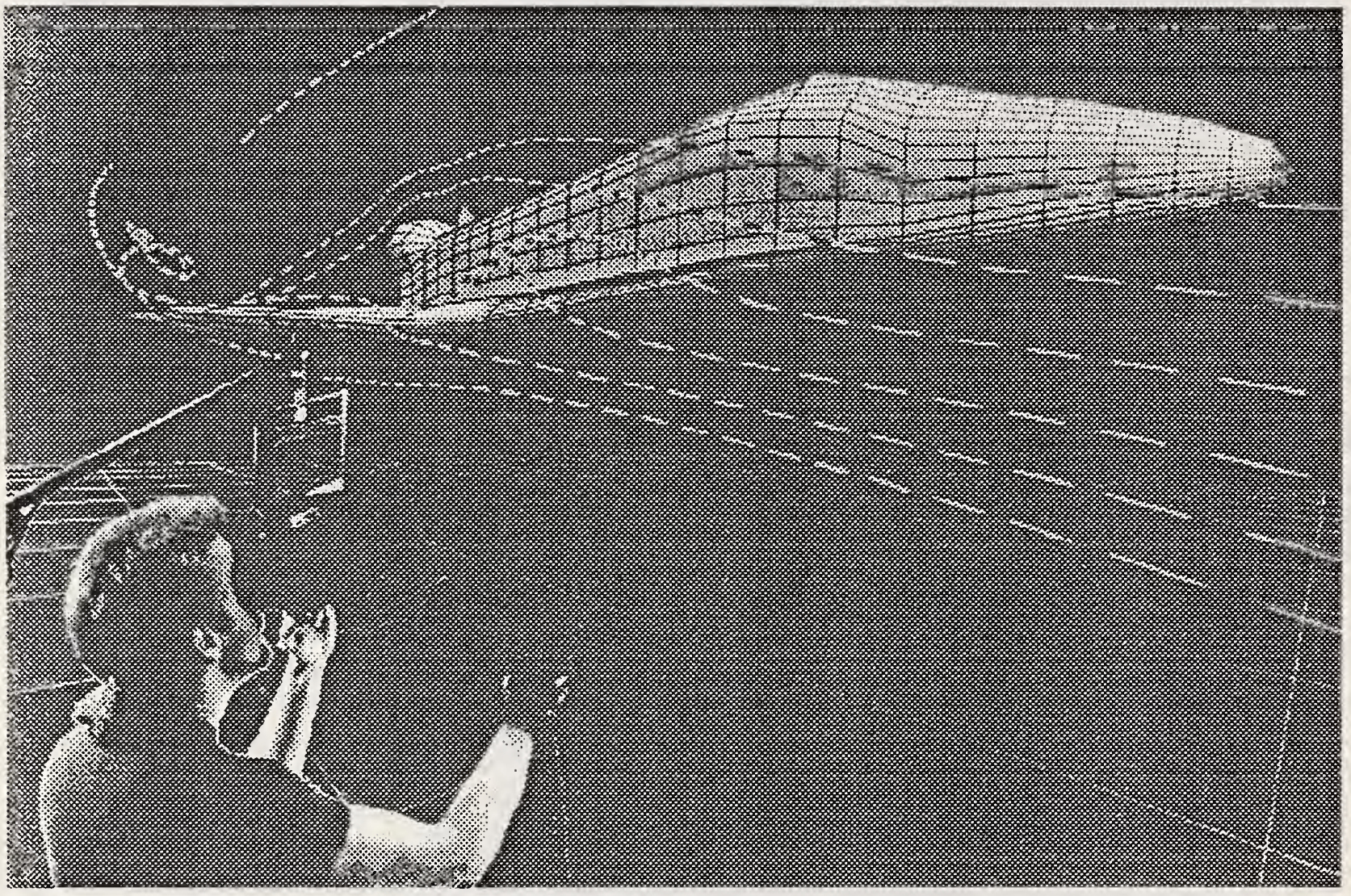

FIGURE 4.

NASA Virtual Wind Tunnel 
As an extension to the original virtual wind tunnel, NASA has continued development by examining feasibility of a distributed virtual windtunnel [Bryson, Gerald-Yamasaki 92]. In this prototype, several users can interact with the data being computed on a Convex C3240 computer and visualized on a Silicon Graphic computer workstation. With the resulting scenario two users can view the same flow visualization and each can observe the flow lines (rakes) injected by the other.

\subsection{Integration Issues}

Integration is perhaps one of the most difficult issues in the domain of VE systems. There are no turn-key solutions to manufacturing problems and very few turn-key VE systems at all. It is difficult, even if you have the money, to simply go out and buy a ready-to-run VE system. One of the only complete systems integrators, VPL, has had major financial problems. The only other complete systems integrator is Division Incorporated, which can provide a fully functional system on a variety of different platforms [DIVISION 93].

One of the significant barriers to the more rapid deployment of complete systems is a lack of standards. There are no interface guidelines for the operation of a motion tracker with a graphics generator or a spatial sound device. As far as VE specifically there are no standards for the representation of VEs which can be used by a variety of VE systems. Many of these issues have been addressed from the CAD domain with standards such as IGES [IGES] and STEP [ISO]; however, the real-time aspects of the data are not part of those standards. Simulation standards and networking protocols such as used in DARPA's SIMNET project [Pope 89], pay a great deal of attention to the networking protocols necessary for real time distributed simulation; however, the detail for the virtual world itself is somewhat lacking.

We can view the need for standards both from a model/world building point of view and from an interface/device point of view. The model/world building applications can draw from the experiences of the CAD domain, and the interface/device issues can draw from the open systems domain.

\subsection{Model/World Building}

Currently the development of a VE is most easily accomplished by using a proprietary package. Products from companies such as Sense8, VREAM and AutoDesk allow users to build (see Appendix B for company addresses) virtual world; however, as in many CAD systems once a model is built with a particular software package you are stuck in that world. Conversion schemes to move a model from one package to another are adhoc at best. Typically custom translators must be built to convert the data.

As far as manufacturing data specifically, the integration of manufacturing data with a virtual environment is no different than the integration of any other type of data with a virtual environment. From a conceptual point of view, however, manufacturing data does have one advantage over purely abstract data: it is usually associated with a physical object. The visualization of a mechanical part, and/or the processes acting on that part is somewhat easier to generate than visualizations of abstract concepts such as 
financial modeling or computational algorithms. This is not to minimize the difficulties of visualizing mechanical processes; however, the physical part does provide a conceptual metaphor that we can mentally grasp.

\subsection{Device Interfaces}

The integration of hardware such as 3D motion trackers, spatial sound devices and head mounted displays also suffers from a lack of standards. As of this writing, there are no standards which would let a vendor write an application that can generically use one of the half dozen or so motion trackers available. The vendor would have to either purchase a library (with the associated licensing costs) or use a VE development environment that supports the required devices. While not an overwhelming problem the lack of interface standards is a barrier to the development of more widespread applications. As an example, there is no standard for $3 \mathrm{D}$ position tracking devices to report their data. This is a barrier to the development of generic software which can take advantage of these technologies.

VE systems require a significant investment in hardware. In order to interface people (to computers, not each other), a wide variety of devices must be integrated into a system. A head-mounted display (HMD) requires miniature displays, a motion tracker requires some I/O to the host computer as does an audio system. There are currently no standards specifically geared towards VE. Device I/O is a major problem. Often for example, a sophisticated 3D motion tracker must be interfaced to the host computer through a slow serial RS-232 line.

The speed of low level device $\mathrm{I} / \mathrm{O}$ is an important factor for VE systems. For example, the typical scenario consists of a user wearing an HMD with a position tracker. The position tracker must send information to the computer which must compute and render new scenes based on the position. If there is a significant lag between the time the user moves his head and the new scene is generated and displayed, the entire immersion effect is lost. Although many factors contribute the overall performance of a system, $\mathrm{I} / \mathrm{O}$ is often a significant problem.

\subsection{Summary}

Virtual environment technology is an evolving collection of technologies. The application of virtual environments to help solve manufacturing problems gives manufacturing engineers new tools to solve complex problems. Virtual environments offer the engineer new ways to not only visualize their problem spaces but to interact with their environment. Many manufacturing problems can be readily visualized by images of part-or manufacturing processes. These visualizations, based on reality, can be used as sf iffective conceptual mechanism for the more complete visualizations embodied by istual environments.

As demonstrated by the variety of case studies in this paper, there are a wi 2 inge of applications for this technology. Two types of applications in the manufactt. - omain which appear to have great potential, particularly for rapid prototyping, ar in nplified by the work at Caterpillar and Ford. Both applications involve a tight integ wion of the 
human into an environment. In the case of Caterpillar, the human is placed into a synthetic environment which represents the prototype of a not-yet-built piece of equipment. The user can observe obstructions and quickly see if there are potential problems with the design. In the Ford application, the motion of the human is the key area under study. In this work the question, "Is an assembly process humanly feasible?" is being studied. In both applications the design process and product development have been sped up by integrating the human being directly into the process.

Although these early applications are highly constrained they are still useful first steps. Decisions made in the design process are the costliest to correct. Virtual environment techniques can improve the design process by integrating human judgement at a far earlier stage of product design then previously possible. Inexpensive changes to the design and rapid prototyping using these environments should improve the quality and reduce the development time for new products.

\subsection{References}

[Autodesk 1993] AutoDesk Press release, Feb 25, 1993, “Autodesk Shops Cyberspace Developers Kit" from sci-vw.

[Bryson, Levit 92] Steve Bryson, Creon Levit, "The Virtual Wind Tunnel," IEEE Computer Graphics \& Applications, July 1992.

[Bryson, Gerald-Yamasaki 92] Steve Bryson, Michael Gerald-Yamasaki, "The Distributed Virtual Windtunnel", in SIGGRAPH 93 Implementing Virtual Reality Course Notes.

[Caudell, Mizell 92] Thomas P. Caudell, David W. Mizell, "Augmented Reality: An Application of Heads-Up Display Technology to Manual Manufacturing Processes" in Proceedings of 1992 IEEE Hawaii International Conference on Systems Sciences, IEEE Press, January 1992.

[Coddington, Chapmen 93] Richard C. Coddington, Dee A. Chapman, "A System for Virtual Reality Simulation of Machinery" to appear in Proceeding of SAE, Milwaukee, Sept., 93.

[Division 93] The Provision 100 Integrated VR System, Division Inc., Suite 101, 400 Seaport Court, Redwood City, CA 94063 (415) 3646067.

[Feiner, MacIntyre \& Seligmann, 92] Steven Feiner, Blair MacIntyre, Doree Seligmann, "Knowledge-based augmented reality" in Communications of the ACM 36(7), July 1993, 52-62. 
[IGES] Initial Graphical Exchange Specification, Version 5.0, IGES/PDES Organization, NTIS PB88235452, National Technical Information Service, Springfield, VA, June 1990.

[ISO] ISO 10303, Standard for The Exchange of Product Model Data, STEP.

[Loftin 1993] R. Bowen Loftin, Virtual Environment Technology for Aerospace Training, Virtual Reality Systems, Vol.1, No. 2 Fall 1993.

[Merner 1992] Jim Merner, Personal communication.

[NCSA 1993] NCSA VR Applications - Virtual Backhoe page of mosaic web, URL: http://www.ncsa.uiuc.edu/Viz/VR/vr_app_cat.html.

[Newquist 1993] Harvey P. Newquist III, AI Expert, Virtual Reality Special Report, 3/93.

[Pope 89] Arthur R. Pope, The SIMNET Network and Protocols, BBN Systems and Technologies Report No. 7102, July 1989

[Sun 92] Demonstration flyer "Sun's Virtual Holographic Workstation Prototype Demonstration," Sun Microsystems Computer Corp., 2550 Garcia Ave. Mountain View, CA 94043, Attn.: Will Shelton, MS PAL1-431, (415) 960-1300 


\section{Appendix A Component Technologies for Virtual Environments}

\section{A.1 Motion Trackers and 3D Input Devices}

One of the essential technologies needed to interact with a virtual environment is a 3D motion trackers. These devices which come in a variety of forms, are used to track the position of a head, a hand, a leg or any object one desires to use as a pointing device. The literature often refers to these devices as six degree of freedom devices because they can report their position in $\mathrm{x}, \mathrm{y}$ and $\mathrm{z}$ and orientation, roll, pitch, and yaw; hence the six degrees of freedom.

Ascension - A Flock of Birds is a six degree of freedom motion tracking device which can be configured to track up to 30 receivers simultaneously. The unit consists of one transmitter and multiple receivers. The flock may be used in an operating range of approximately 10 feet.

Ascension Technology Corp.

P.O. Box 527

Burlington, VT 05402

(802) 655-7879

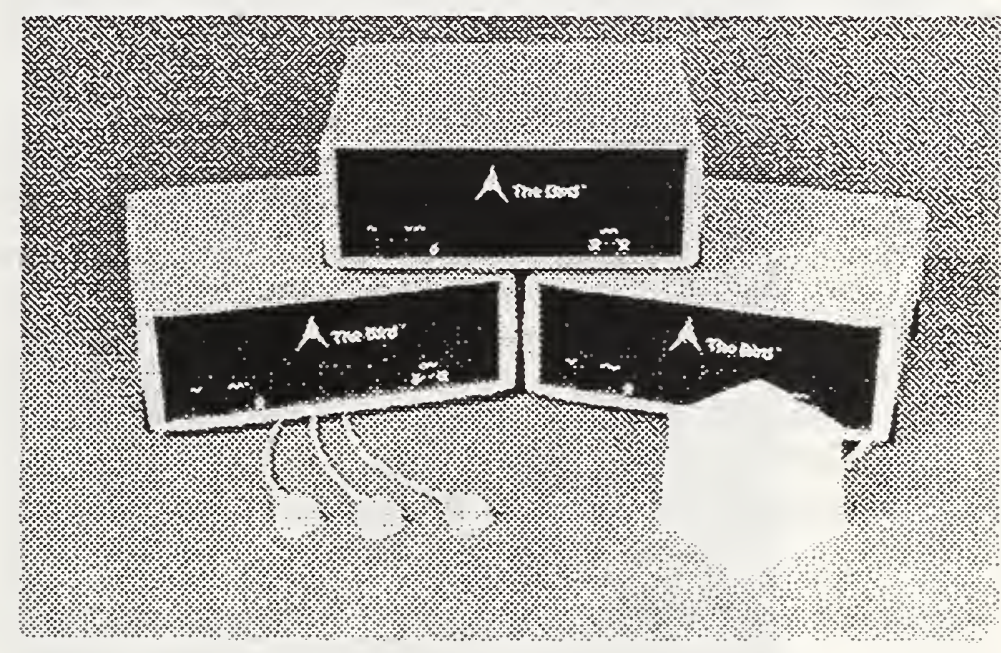

FIGURE 5.

Ascension's Flock of Bird (illustrated with 3 transmitters) 
Logitech Inc.

6505 Kaiser Drive

Fremont, CA 94555

(510) $795-8500$
Logitech 3D Mouse - The Logitech 3D Mouse functions as a standard 2D mouse or as a 3D mouse which can report position, and orientation (roll, pitch, and yaw). A triangular array of 3 ultrasonic speakers sends signals to a triangular set of microphones on the mouse that samples the signals at up to 50 times per second. The mouse and triangle communicate along a line of sight and both the mouse and triangle array are connected to a control unit.

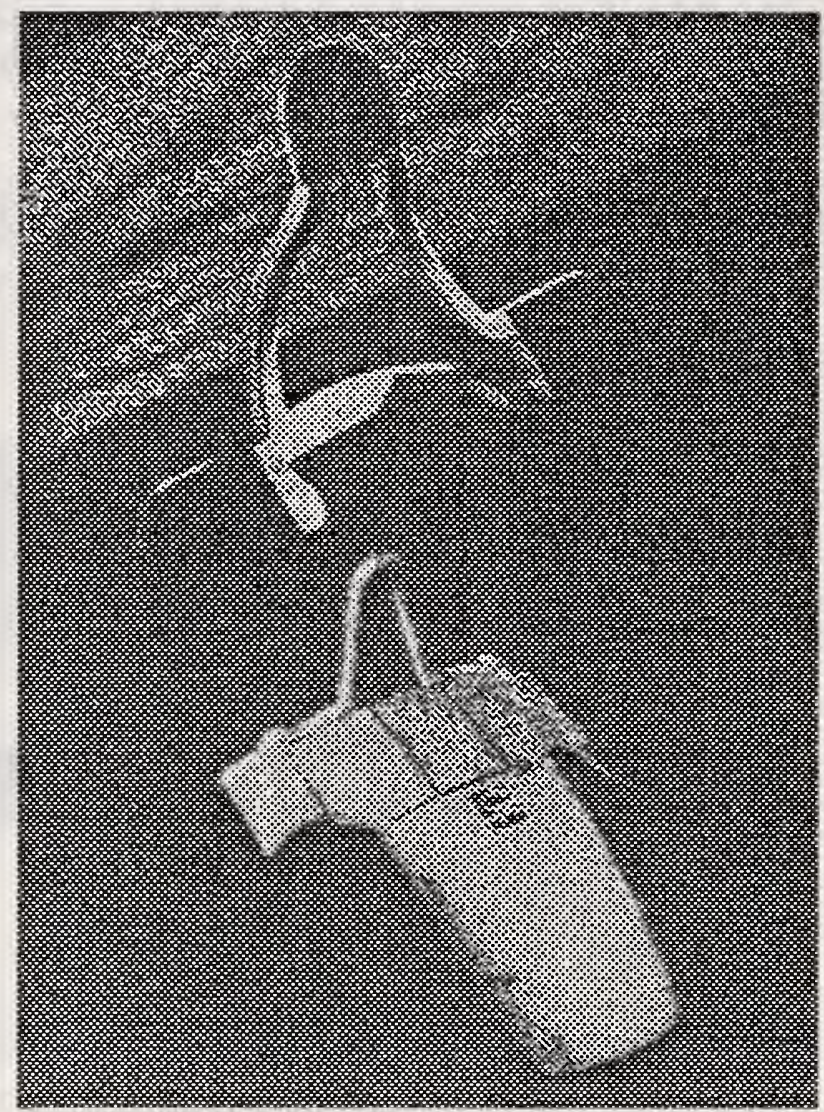

Logitech 3-D mouse

FIGURE 6. 
Polhemus, Inc.

P.O. Box 560,

1 Hercules Drive, Colchester, VT 05446

(802) 655-3159

\section{FIGURE 7.}

Spatial Systems, inc.

Middlesex Technology Center, 900 Middlesex Turnpike, Building 8 ,

Billerica, MA 01821
Polhemus - Polhemus Systems offers a 3D position tracking systems based on low-frequency magnetic fields. Their FastTrak product is a widely used solution for reliable position tracking.

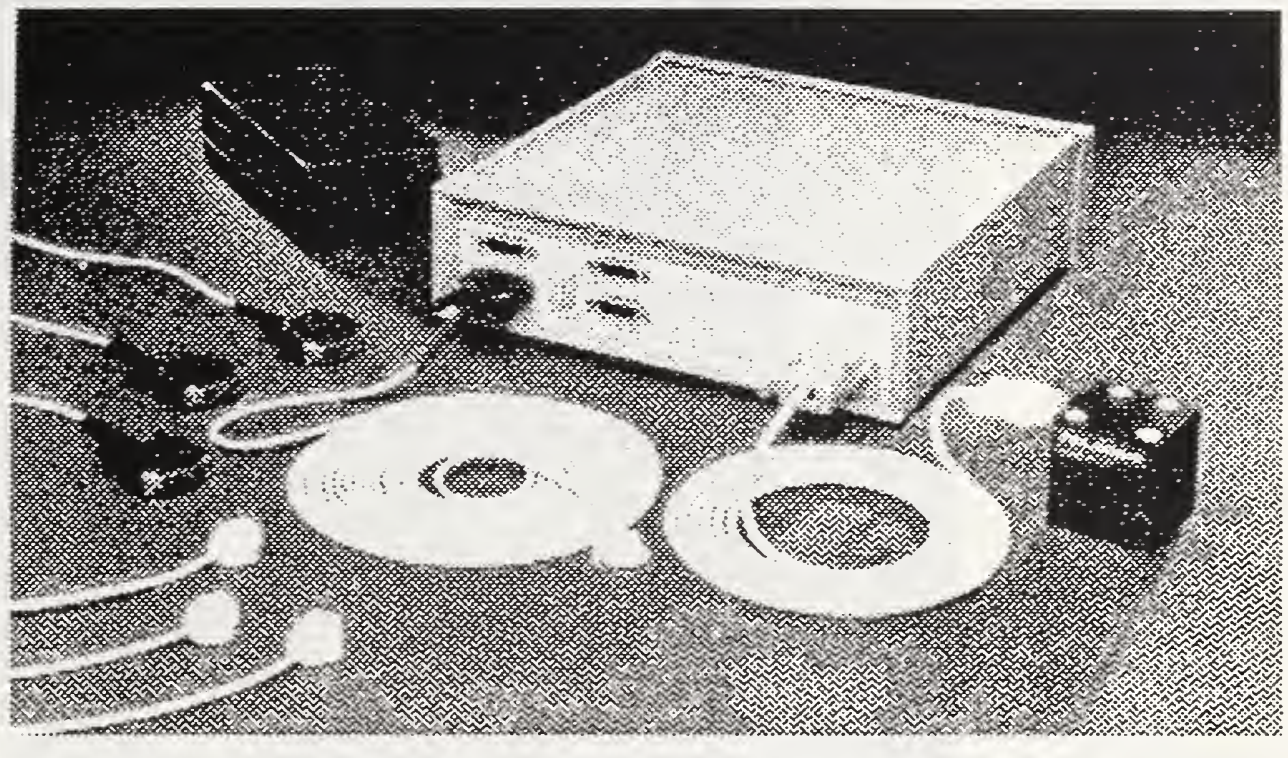

\section{Polhemus FASTRAK}

The Spaceball is a 3-D control device which looks like a billiard ball mounted on a short stick which can sit next to a computer. Inside the ball is a 6 degree of freedom sensor which can be used very effectively to position and orient object or to travel over some data terrain. Although not typically thought of as a 3D tracking device the Spaceball is very practical for the manipulation of objects in a conventional computing environment.

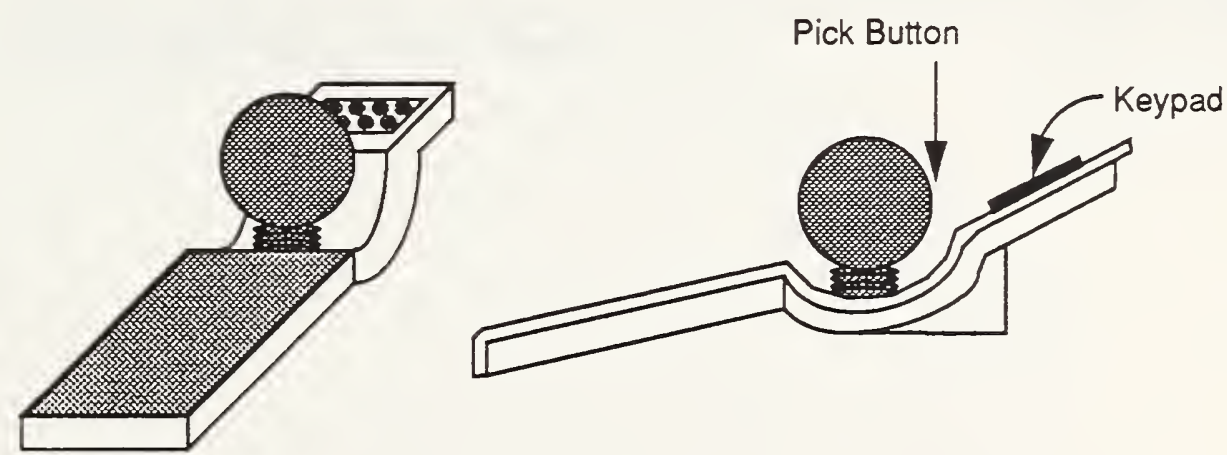

FIGURE 8.

Diagram of Spaceball 
EXOS, Inc.

8 Blanchard Road

Burlington, MA 01803

(617) 229-2075
EXOS, Inc. has a high-precision exoskeleton device that is worn like a glove. The "Dexterous Hand Master" can track finger-joint angles with a high degree of resolution.

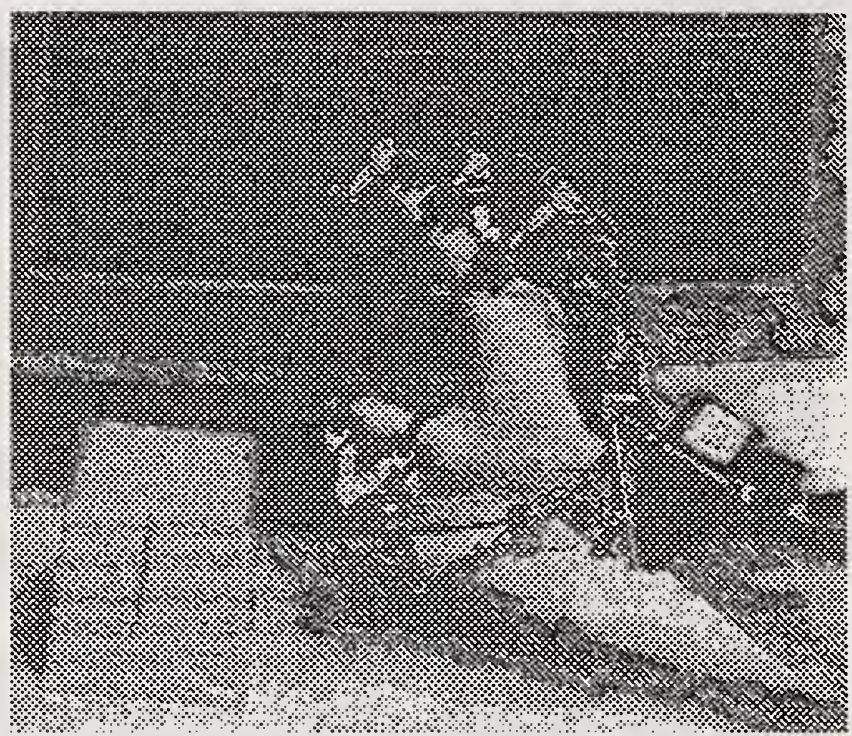

Dextrous Hand Master (with 3D position tracker)

FIGURE 9.

\section{A.2 Display Technology}

One critical element required to achieve immersion into a virtual environment is the ability to adjust a display while tracking the position of the head. The computer display must remain in front of your eyes, even when your head moves. This is why helmets and other display devices that move with you have come into existence. 


\section{A.2.1 3D Devices}

CrystalEyes is a LCD based 3D display system. The user wears a set of LCD glasses powered by batteries, so that there are no wires. A transmitter which sits on top of the monitor of a workstation synchronizes the LCD shutter glasses with the computer display, generating 3D images.

StereoGraphic Corporation 2171-H East Francisco Boulevard San Rafael, CA 94901 (415) $459-4500$

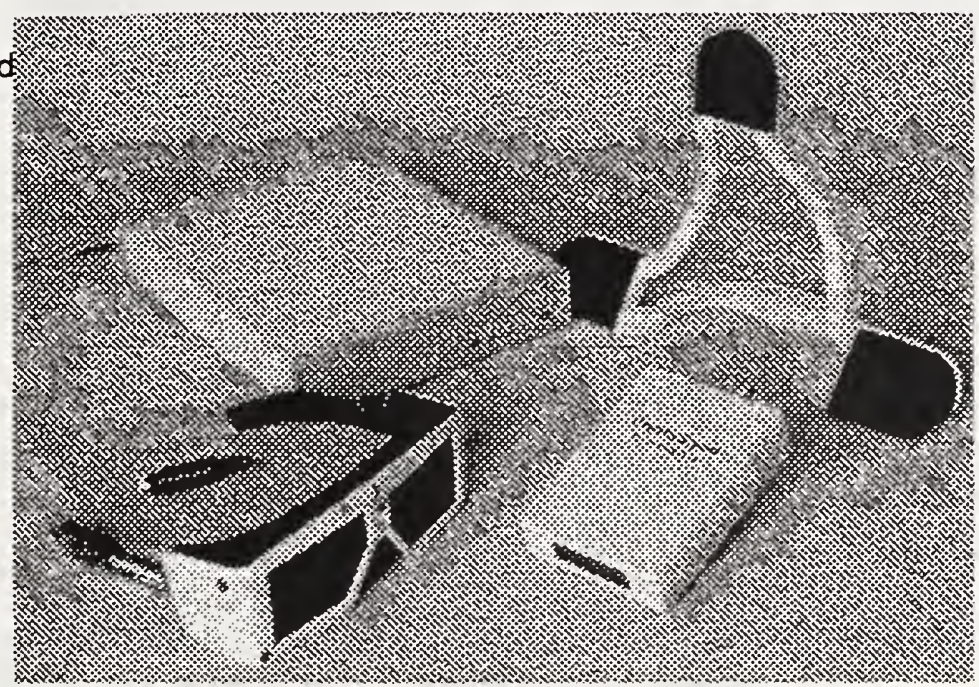

FIGURE 10

StereoGraphics CrystalEyes 3D display system

\section{A.2.2 Booms}

A boom is a display device, a CRT, mounted on a support that counterbalances the weight of the display. Booms can be configured either as monocular or stereo devices. One of the principal advantages of booms is that they can support high resolution CRT's not typical of the Head Mounted Displays. 
Fake Space Labs 935 Hamilton Ave. Menlo Park, CA 94025 (415) 688-1940

FIGURE 11.

LEEP Systems Inc., 241 Crescent St., Waltham, MA 02154 (617) 657-1395
Fake Space Labs offers a number of booms which incorporate both high resolution CRT's and motion tracking. They can provide either black and white or color displays.

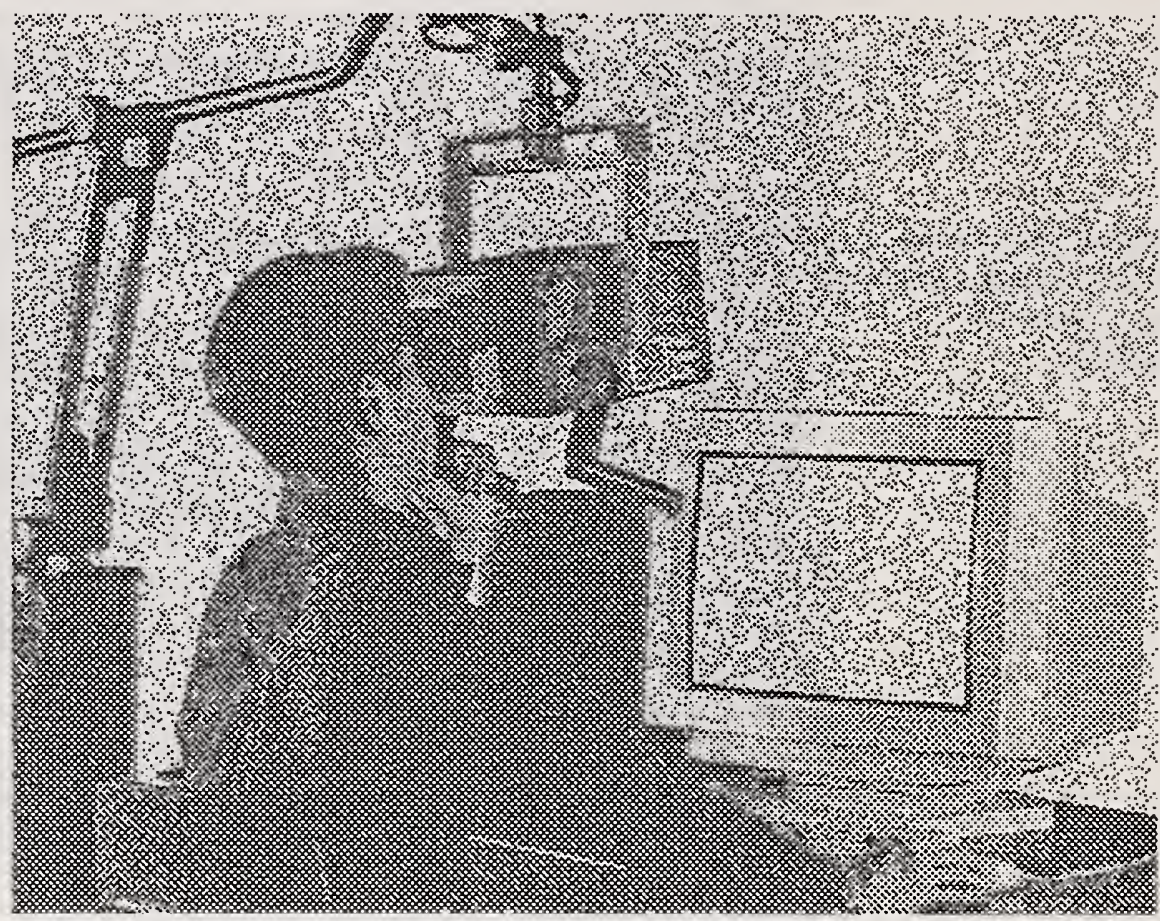

The Fake Space Labs Boom

LEEP Systems offers the CYBERFACE3 system, a relatively low cost $(\sim 10,000)$ boom type display system.

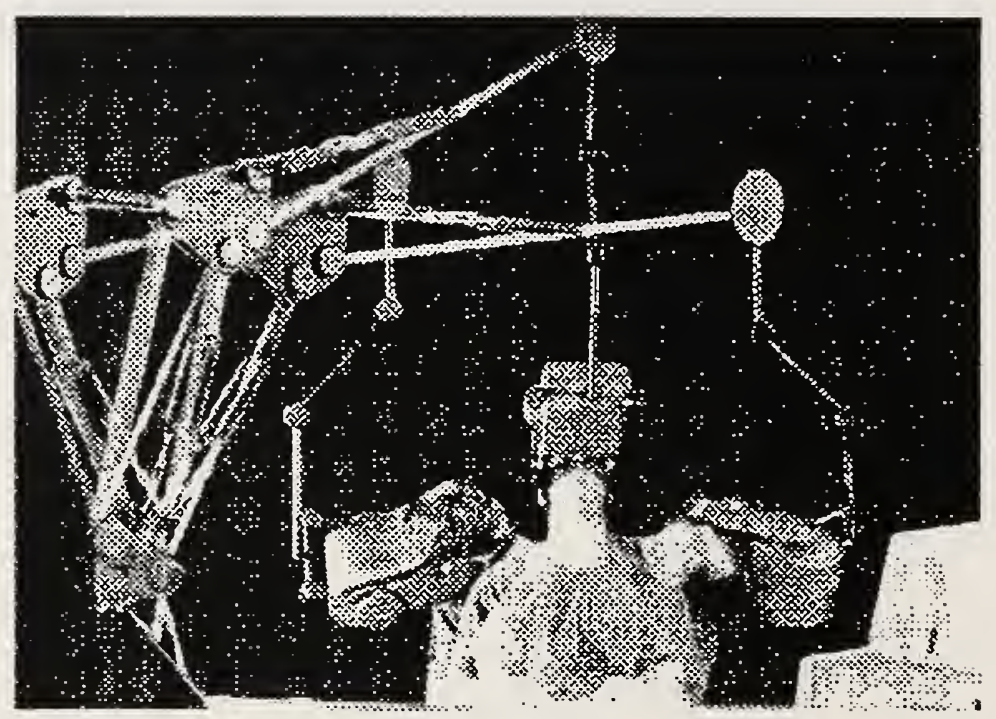

LEEP Systems Cyberface 3 


\section{A.2.3 Head Mounted Display (HMD)}

$\mathrm{n}$-Vision, Inc. offers some of the highest quality head-mounted displays available. Their recent product is the Datavisor 9c which can display $1280 \times 960$ stereo color images. High resolution color CRTs are mounted in a helmet.

$n$-Vision Inc.

800 Follin Lane, Suite 270

Vienna, VA 22180

(703) $242-0030$

\section{FIGURE 13.}

Virtual Research 3193 Belick St., Suite \#2 Santa Clara, CA 95054 (408) $748-8712$

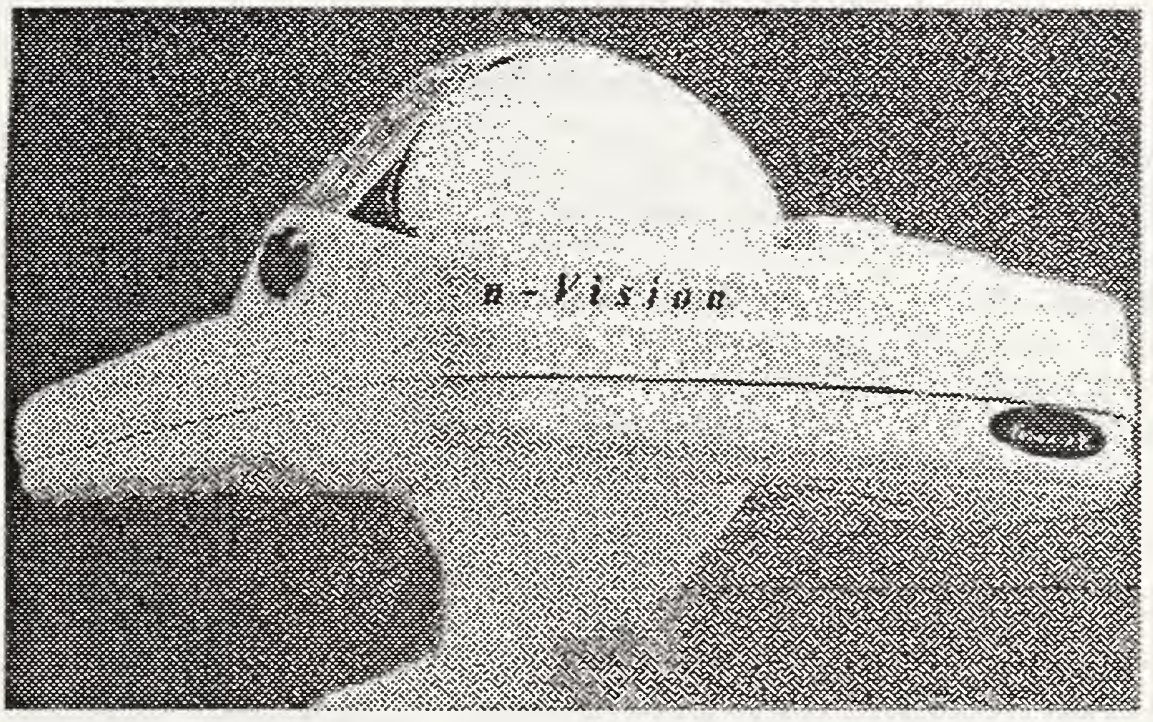

Datavisor $9 \mathrm{C}$ high resolution helmet mounted display

Virtual Research offers the Eyegen 3 which can display a medium resolution color images, mounted in a helmet. The Eyegen contains 2 medium resolution black and white CRT which are housed with spinning color wheels to generate the color.

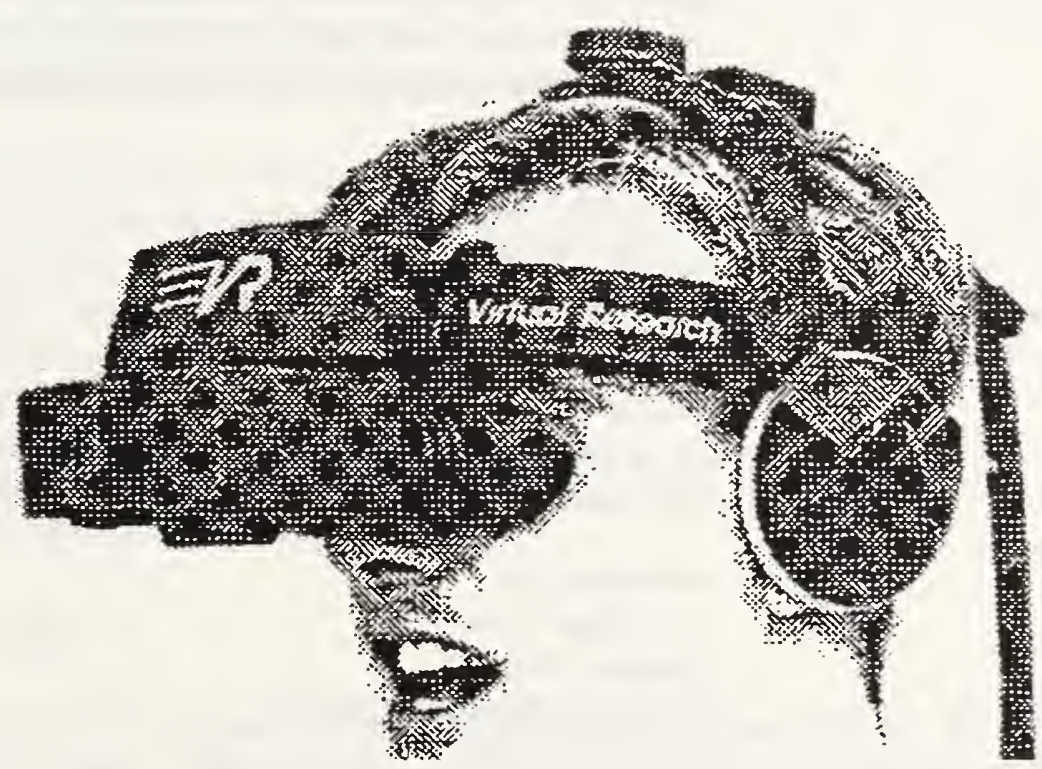

The Virtual Research Eyegen3

FIGURE 14. 


\section{A.3 Spatial Audio}

Audio, associated in three dimensions with objects can add a great deal to the sensation of immersion. Devices that can produce effective aural environments are available from a number of sources, a sampling is listed below.

The Convolvotron is a PC based board, and is a serious tool for the study and manipulation of sound in $3 \mathrm{D}$.

Crystal River Engineering

12350 Wards Ferry Road

Groveland, CA 95321

(209) $962-6382$

A 3D audio device for high end workstations such as Silicon Graphics is the SoundCube by VSI.

Visual Synthesis, Inc.

4126 Addison Rd.

Fairfax, VA 22030

(703) $352-0258$

\section{A.4 Force Feedback Devices}

Force feedback devices give the user the sensation feedback when their virtual pointer or hand "touches" a virtual object. The idea is to let the user know, for example, that their hand has grasped an object. This is an extremely immature technology when it comes to use in virtual environments, however, bulky devices for use in teleoperation and telerobotics are somewhat more mature and effective for particular remote applications.

Sarcos Inc. manufactures the Sarcos Dextrous arm, a force reflexive device in which you place your arm and another robotic arm moves in the same manner. Although it was designed for use in teleoperation applications it may be useful for virtual environment applications.

Sarcos Incorporated, 261 East 300 South, Suite 150

Salt Lake City, UT 84111

(801) 531-0559

Another interesting device is a glove with small air lines which provide pressure at appropriate times. The device can both sense and record pressure allowing objects to be "felt" according to a previously sampled pressure.

Airmuscle Ltd.

12 Orchard Close

Cranfield, Bedford

United Kingdom MK43 OHY

(44) 234-750-791 


\section{Appendix B Off-the-shelf Software components}

The following describes a number of software libraries and applications expressly designed for the development of virtual environments. Most are $\mathrm{C}$ or $\mathrm{C}++$ libraries which can be integrated into custom applications and all support the various required input and output user interface devices.

\section{B.1 Autodesk Cyberspace Developers Kit}

Autodesk, developers of the AutoCAD series of products, has since May 1993 offered $\mathrm{CDK}$, the Cyberspace Developers Kit. CDK consists of $\mathrm{C}++$ class libraries designed to facilitate the development of virtual environments on 386 or 486 platforms. CDK includes support for a large number of peripherals including head-mounted displays, glove devices and position trackers. According to Richard Dym, general manager of Autodesk's Multimedia Division, "We at Autodesk view Virtual Reality as the logical next step in design automation, because it adds interactive 3D visualization and simulation capabilities to the drafting and design process." [Autodesk 1993] CDK lists for $\$ 2,495$. For more information on Autodesk call 1-800-879-4233

\section{B.2 SENSE8 WorldToolKit}

SENSE8's WorldToolKit 2.0 is a set of $C$ functions that is designed to run across a number of platforms including PC's and Silicon Graphics workstations. WorldToolKit contains support for most of the popular input and output devices

SENSE8,

4000 Bridgeway Suite 101,

Sausalito, CA 94965

(415) 331-6318

\section{B.3 University of Alberta Minimal Reality (MR) Toolkit}

The MR toolkit is a university developed subroutine library available with source code that supports the development of virtual reality interfaces. MR provides support for the Polhemus and Ascension motion trackers, VPL DataGlove, VPL EyePhone. The current version of MR is callable from C and FORTRAN on SGI, RS6000, and DEC workstations. Unlike typical university projects, there is an extensive amount of documentation the code is actually documented.

MR is available at no cost for research purposes by sending email to: mr-help@cs.ualberta.ca

\section{B.4 VREAM}

The VREAM Virtual Reality Development System from VREAM, Inc. is a low cost PC based system. VREAM offers an application called the VREAM 3D World Editor and the VREAM Scripting Language as mechanism to build and edit virtual worlds. The 3D 
objects can be controlled and manipulated using the VREAM Runtime System and there is support for 3D motion trackers, glove, and helmet-mounted displays. VREAM lists for $\$ 795$.

VREAM Inc.

2568 N. Clark St. \#250

Chicago, IL 60614

(312) 477-0425 


\section{Appendix C Sources of Information}

\section{C.1 Books}

Aukstakalnis, S. \& Blatner, D. (1992). Silicon Mirage: The Art and Science of Virtual Reality. Peachpit Press, Berkeley, California.

An excellent book introducing all of the relevant technologies that are used to create virtual reality systems. Best of all the hype so often found in the press is left out. This is a serious collection of useful information and background on VR.

Blattner, M. \& Dannenberg, R. B., eds. (1992). Multimedia Interface Design. ACM Press/Addison-Wesley, Reading, MA A collection of good papers about multimedia design including several relevant to the individual technologies that go into VR systems. These include haptic feedback, sound and novel metaphors.

Krueger, M. W. (1991). Artificial Reality II. Addison-Wesley, Reading, MA.

Provides some good background on the origins and early "classic" works in VR. Tends to drift into the more philosophical and idiosyncratic views of the author but is still worthwhile.

Laurel, B. (1991).Computers as Theater. Addison-Wesley, Reading, MA. Primarily a book about a new metaphor for thinking about user interfaces, that is applicable to VR. Includes a section specifically about VR and discusses, in several places, how VR and user interaction are related.

\section{C.2 Networks}

Compuserve contains a VR section which may be accessed though the COMART forum by typing GO COMART in the Compuserve information manager.

USENET newsgroup, sci.virtual-worlds is a moderated newsgroup containing discussions and announcements of the latest VR topics.

\section{C.3 Periodicals}

CyberEdge Journal, \#1 Gate Six Road, Suite G, Sausalito, CA 94965 A bimonthly newsletter of up to date VR industry information. (\$129/year, six issues) 
IRIS Universe, A quarterly magazine for uses of Silicon Graphics computer which usually has good articles about VR. Silicon Graphics 2011 North Shoreline Blvd. Mail Stop 415, Mountain View, CA 94039-7311 (free to qualified users).

PRESENCE: Teleoperators and Virtual Environments, published by The MIT Press, Cambridge, MA 02142

A quarterly refereed journal of high quality articles.

Virtual Reality Report, published by Meckler Corp. 11 Ferry Lane West, Westport, CT 06880 (203) 226-6967. (\$277 / year - ten issues). Newsletter of current events and happening in the VR world. Good coverage of conferences and a section devoted to VR in Europe.

SophisTech Research (1992). Virtual Reality Sourcebook, SophisTech Research, 6936 Seaborn St., Lakewood CA, 90713-2832, (800) 4VR-SOURCE.

A comprehensive reference of virtually everything and anything having relevance to the VR industry. Is sold as a loose leaf collection of pages, which unfortunately does not contain an index. $\$ 129$ and a IBM-PC electronic version is available for $\$ 95$. Quarterly updates for $\$ 25$ ea. 
